\title{
RAMIFICATION IN ELLIPTIC MODULAR FUNCTION FIELDS
}

\author{
DONALD L. MCQUILLAN
}

1. The field of elliptic modular functions of level $n$ is a finite galois extension $K$ of the field $\mathbf{C}(j)$ generated over $\mathbf{C}$ by the Weierstrass absolute invariant $j$. Furthermore, the galois group is $\operatorname{LF}(2, n)$ $=\mathrm{SL}(2, Z / n Z) / \pm I_{2}$ and the genus $g$ of $K$ is given by

$$
2 g-2=1 / 12 \cdot(n-6) n^{2} \prod_{q \mid n}\left(1-1 / q^{2}\right)
$$

for $n>2$ ( $g=0$ for $n=1,2$ ). If $p$ is a prime number not dividing $n$ and if $k$ is an algebraic closure of $\mathrm{GF}(p)=Z / p Z$ ( $k$ can also be an algebraic closure of $\mathbf{Q})$, Igusa [4] constructed a galois extension of $k(j)$ with the same galois group and the same genus. On the other hand, if the level $n$ is a prime number $q$, Hecke [3] proved that $K / \mathbf{C}(j)$ is uniquely determined by the two properties. The purpose of this short note is to extend this theorem of Hecke in the following way:

Theorem. Let $K / k(j)$ be a galois extension of genus $g(n=q)$ with $\mathrm{LF}(2, q)$ as galois group. Then, the ramification of $K / k(j)$ is uniquely determined. Hence, (as in Igusa's extension) $K / k(j)$ is ramified over one point with index $q$ and over two other points with indices 2, 3 for $p \neq 2,3$, over one other point with the tetrahedral group as inertia group (second ramification group $=$ trivial) for $p=2$ and with the dihedral group of order 6 as inertia group (second ramification grou $p=$ trivial) for $p=3$. Moreover, in the case $p \neq 2,3$, (if we fix three points with ramification indices $2,3, q)$ the extension $K / k(j)$ is uniquely determined.

2. We shall start proving the theorem. Since the case $q=2$ can be treated separately (and rather easily), we shall assume that $q$ is an odd prime. Suppose that $K$ is ramified over $k(j)$ at $j=a_{1}, a_{2}, \cdots, a_{w}$ and that

$$
T\left(a_{i}\right) \supset V_{1}\left(a_{i}\right) \supset V_{2}\left(a_{i}\right) \supset \cdots
$$

is a sequence of the inertia group and the first, second, $\cdots$ ramification groups at a place of $K$ lying over $a_{i}$. Then, it is a normal sequence (unique up to an inner automorphism of $\operatorname{LF}(2, q)$ ) such that $V_{1}\left(a_{i}\right)$ is the unique $p$-Sylow group of $T\left(a_{i}\right)$ with cyclic factor group. In particular, the commutator group of $T\left(a_{i}\right)$ has to be a $p$-group. Now, thanks to Gierster [1], we know all subgroups of $\operatorname{LF}(2, q)$ : A subgroup of $\operatorname{LF}(2, q)$ is

Received by the editors March 26, 1962. 
(i) a cyclic group $C_{m}$ of order $m$ where $m=q, m \mid(q-1) / 2$ or $m \mid(q+1) / 2$,

(ii) a dihedral group $D_{2 n}$ of order $2 n$ where $n \mid q-1$ or $n \mid q+1$,

(iii) a metacyclic group of order $q t$ where $t \mid(q-1) / 2$ with $C_{q}$ as commutator group, or

(iv) a tetrahedral, octahedral or icosahedral group. Because of the property of $T\left(a_{i}\right)$ mentioned above, candidates for $T\left(a_{i}\right)$ are limited. In fact, they are $C_{m}$ in (i), $D_{2 n}$ in (ii) with $n=p^{r}$ ( $p$ odd) and the tetrahedral group. This being remarked, the "relative genus formula" applied to $K / k(j)$ gives

$$
\sum_{i}\left(E_{i}-1\right) / e_{i}=(2-w)+1 / 6-1 / a
$$

where

$$
\begin{aligned}
e_{i} & =\operatorname{ord} . T\left(a_{i}\right), \\
E_{i} & =\left(\operatorname{ord} . V_{1}\left(a_{i}\right)-1\right)+\left(\operatorname{ord} . V_{2}\left(a_{i}\right)-1\right)+\cdots
\end{aligned}
$$

Since the right side of the genus formula is not integral at $q$, at least one $e_{i}$, say $e_{1}$, is a multiple of $q$. Then $T\left(a_{1}\right)$ is either $C_{q}$ or the tetrahedral group (with $q=3, p=2$ ). In the second case, $K$ contains a cyclic subextension of $k(j)$ of degree 3 , hence $e_{2}$, say, is also a multiple of $q=3$. If $T\left(a_{2}\right)$ is again the tetrahedral group, we get $e_{1}=e_{2}=12$, $E_{1}, E_{2} \geqq 3$, and this will bring a contradiction. Hence, we can always assume that $T\left(a_{1}\right)$ is $C_{q}$. This implies

$$
\sum_{i>1}\left(E_{i}-1\right) / e_{i}=(2-w)+1 / 6
$$

Since $e_{i} \geqq 2$ and $E_{i} \geqq 0$, therefore, we have $w \leqq 3$ and certainly $w \geqq 2$. Suppose, first, that $w=3$. Then, we see immediately that $e_{2}=2$. $e_{3}=3$ with $E_{2}=E_{3}=0$, hence $p \neq 2$, 3. Suppose, next, that $w=2$, Then, we have $e_{2}=6\left(E_{2}-1\right)$ and this is a multiple of $p$. Consequently, $T\left(a_{2}\right)$ is $C_{m}$ in (i), $D_{2 n}$ in (ii) with $n=3^{r}$ or the tetrahedral group (with $\left.V_{2}\left(a_{2}\right)=1\right)$. In the second case, we see that $T\left(a_{2}\right)=D_{6}\left(\right.$ with $\left.V_{2}\left(a_{2}\right)=1\right)$. We shall show that the first possibility has to be rejected entirely.

3. We recall [1] that subgroups in (i), (iii) are unique up to inner automorphisms of $\operatorname{LF}(2, q)$. We denote by $\Sigma$ the subextension of $k(j)$ which corresponds (by the theory of Galois) to the group of linear transformations $x \rightarrow a^{2} x+b$ with $a$ in $\mathrm{GF}(q)^{*}$ (=multiplicative group of $\mathrm{GF}(q))$ and $b$ in $\mathrm{GF}(q)$. Using Hilbert's galois theory, we shall calculate the relative genus formulas for $K / \Sigma$ and for $\Sigma / k(j)$ (cf. [4, pp. 473-474]). In doing this, we can assume that $T\left(a_{1}\right)$ is the group of 
linear transformations $x \rightarrow x+b$ with $b$ in $\mathrm{GF}(q)$. Suppose, first, that $T\left(a_{2}\right)=C_{e_{2}}$ with $e_{2} \mid(q-1) / 2$. Then, we can assume that $T\left(a_{2}\right)$ is the subgroup of order $e_{2}$ of the group of linear transformations $x \rightarrow a^{2} x$ with $a$ in $G F(q)^{*}$. Thus, if $g_{0}$ is the genus of $\Sigma$, we get

$$
\begin{aligned}
& 2 g-2=(q-1)^{2} / 2+q(q-1) / e_{2} \cdot\left(\left(e_{2}-1\right)+E_{2}\right)+q(q-1) / 2 \cdot\left(2 g_{0}-2\right), \\
& 2 g_{0}-2=(q-1)+(q-1) / e_{2} \cdot\left(\left(e_{2}-1\right)+E_{2}\right)-2(q+1) .
\end{aligned}
$$

By eliminating $g_{0}$, we get $q(q+1)(q-1)=0$. This is a contradiction. Suppose, next, that $T\left(a_{2}\right)=C_{e_{2}}$ with $e_{2} \mid(q+1) / 2$. Then, in the same way we get

$$
\begin{aligned}
2 g-2 & =(q-1)^{2} / 2+q(q-1) / 2 \cdot\left(2 g_{0}-2\right), \\
2 g_{0}-2 & =(q-1)+(q+1) / e_{2} \cdot\left(\left(e_{2}-1\right)+E_{2}\right)-2(q+1),
\end{aligned}
$$

and hence $q(q+1)(q-1)=0$. This is a contradiction.

4. Finally, we shall indicate how the uniqueness of $K / k(j)$ follows from the information about ramifications in the case $p \neq 2,3$. Suppose that $K_{1} / k(j), K_{2} / k(j)$ are two such extensions, i.e. with the same genus $g$ and with $L F(2, q)$ as galois group. By an automorphism of $k(j)$, we can make an adjustment so that $K_{1} / k(j), K_{2} / k(j)$ are ramified over the same three points $a_{1}, a_{2}, a_{3}$ with the same indices. Consider their compositum $\Omega / k(j)$ (in some algebraic closure of $k(j)$ ). Then $\Omega / k(j)$ is ramified only over $a_{1}, a_{2}, a_{3}$ and, in fact, tamely. Let $G$ be the galois group of $\Omega / k(j)$ and let $H_{1}, H_{2}$ be the normal subgroups of $G$ which correspond to $K_{1}, K_{2}$. Then, by a general result of Grothendieck [2], we can pick $\sigma_{1}, \sigma_{2}, \sigma_{3}$ from inertia groups over $a_{1}, a_{2}, a_{3}$ which generate $G$ and which satisfy $\sigma_{1} \sigma_{2} \sigma_{3}=1$. Let $\sigma_{i}^{\prime}, \sigma_{i}^{\prime \prime}$ be the images of $\sigma_{i}$ in $G / H_{1}, G / H_{2}$. Then, by a lemma of Hecke [3, p. 574], there exists an isomorphism $G / H_{1} \simeq G / H_{2}$ in which $\sigma_{i}^{\prime}$ and $\sigma_{i}^{\prime \prime}$ correspond to each other. This is possible (if and) only if $H_{1}=H_{2} \mathrm{com}$ pleting the proof.

\section{REFERENCES}

1. J. Gierster, Die Untergruppen der Galois'schen Gruppe der Modulargleichungen für den Fall eines primzahligen Transformation-grades, Math. Ann. 18 (1881), 319-360.

2. A. Grothendieck, Géométrie formelle et géometrie algêbrique, Seminaire Bourbaki, May, Secrétariat Mathématique, Paris, Exposé 182, 1959.

3. E. Hecke, Die eindeutige Bestimmung der Modulfunktionen q-ter Stufe durch algebraische Eigenschaften, Collected Works, Vandenhoeck and Ruprecht, Göttingen, 1959, pp. 568-576.

4. J. Igusa, Fibre systems of Jacobian varieties. III, Fibre systems of elliptic curves, Amer. J. Math. 81 (1959), 453-476.

University College Galway, Ireland 